INPLASY

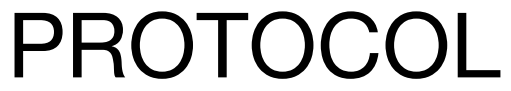

To cite: Zeng et al.

Complementary and

Alternative Therapies of 7 Non-

pharmaceutical Means for

Anxiety: A Systematic Review

and Network Meta-analysis.

Inplasy protocol 202070069.

doi:

10.37766/inplasy2020.7.0069

Received: 16 July 2020

Published: 16 July 2020

Corresponding author:

Bingxue Liang

liangyilan_gloria@163.com

Author Affiliation:

Chongqing Medical University

Support: cstc2019jcyj-

msxmX0180.

Review Stage at time of this submission: The review has not yet started.

Conflicts of interest:

We declare that we do not have any commercial or associative interest that represents a conflict of interest in connection with the work submitted.

\section{Complementary and Alternative Therapies of 7 Non-pharmaceutical Means for Anxiety: A Systematic Review and Network Meta-analysis}

Zeng, J1; Zheng, Z²; Chen, Z3; Liang, B4.

Review question / Objective: To analyze and compare the effects of various complementary therapies on anxiety disorders.

Condition being studied: Anxiety disorders.

Information sources: We performed a literature search for published and unpublished randomized clinical trials in four English databases (Medline, Embase , Cochrane Library, and Web of Science) and three Chinese databases (China National Knowledge Infrastructure [CNKI], Weipu and Wanfang) from inception until July 15, 2020.

INPLASY registration number: This protocol was registered with the International Platform of Registered Systematic Review and Meta-Analysis Protocols (INPLASY) on 16 July 2020 and was last updated on 16 July 2020 (registration number INPLASY202070069).

\section{INTRODUCTION}

Review question / Objective: To analyze and compare the effects of various complementary therapies on anxiety disorders.
Condition being studied: Anxiety disorders.

\section{METHODS}

Participant or population: Inclusion: There are no restrictions on the age, race, gender, 
anxiety level and duration of the disease of patients who meet the diagnostic criteria of anxiety disorder and anxiety state organized by various societies. Exclusion: Anxiety disorder with personality division and other congenital psychosis.

Intervention: The experimental group adopted one of seven common non-drug therapies: acupuncture, moxibustion, massage, aromatherapy, cognitive therapy, music therapy, and visual therapy.

Comparator: The intervention measures of the control group include: blank, placebo, basic treatment, diet therapy, one of the five control methods.

\section{Study designs to be included: RCT.}

Eligibility criteria: In this study, only randomized controlled trials were included, and 2 or more control groups were required. Do not strictly limit the randomized methods, or just mention "randomization" in the methods section, whether it is detailed or not. Due to the particularity of non-drug therapy, it is difficult to implement the strict doctorpatient "double blind method" in most cases, so it can be included whether the blind method is implemented or not.

Information sources: We performed a literature search for published and unpublished randomized clinical trials in four English databases (Medline, Embase , Cochrane Library, and Web of Science) and three Chinese databases (China National Knowledge Infrastructure [CNKI], Weipu and Wanfang) from inception until July 15, 2020.

Main outcome(s): (1)State-Trait Anxiety Inventory (STAI); (2)Visual Analogue Scaleanxiety (VASA); (3)Hamilton Anxiety Scale (HAMA).

Additional outcome(s): (1)Blood pressure systolic blood pressure; (2) heart rate; (3)Hamilton Depression Scale (HAMD); (4)Numerical Rating Scale (NRS).
Quality assessment / Risk of bias analysis: The two reviewers independently assessed the methodological quality of the randomized controlled clinical studies through the Revman software provided by the Cochrane Collaboration and the RISK OF BIAS tool. In case of differences, the third reviewers ruled. The content of the assessment is referred to the Cochrane Handbook for Systematic Reviewers, including the following seven aspects: 1 . Whether the random method is correct; 2. Whether the group is hidden, and whether the hidden method is fought for; 3 . Whether the blind method is adopted, and whether the blind method is correct; 4 . Whether to use blind method for outcome efficacy evaluation and statistical treatment; 5 . Whether to report the number of dropouts and departures and their reasons; 6 . Selective reporting; 7. Other deviations (conflicts of interest, etc.). The assessment risk levels are divided into "low" (low risk), "high" (high risk) and "unclear".

Strategy of data synthesis: The data were analyzed by odds ratio (OR). The measurement data were analyzed by weighted mean difference (WMD) and 95\% confidence interval $(95 \% \mathrm{Cl})$. If the mean and standard deviation of continuous variables before and after intervention were not reported in the literature, they were converted according to the formula provided by the Cochrane Collaboration. The $U$ test was used for statistical tests, and the results were expressed by $Z$ and $P=0.05$, there was a statistically significant difference in the efficacy of different treatment regiments.

Subgroup analysis: No subgroup analysis is involved.

Sensibility analysis: The heterogeneity of the effect size was evaluated using tausquared statistics. A fixed effect model was used when the tau-squared value was $<50 \%$; otherwise, a random effects model was used. We used funnel plots to investigate the publication bias in our meta-analysis. To explore the sources of heterogeneity, we performed a meta- 
regression analysis with weight dependence.

Country(ies) involved: All countries whose results were retrieved and published in Chinese or English were included.

Keywords: Complementary and Alternative Therapies, anxiety, network meta analysis.

Contributions of each author:

Author 1 - Juan Zeng.

Author 2 - Zhuanfang Zheng.

Author 3 - Zhiwei Chen.

Author 4 - Bingxue Liang. 